\title{
Sintomas vestibulares em crianças com queixa de dificuldades escolares
}

\section{Vestibular symptoms in children with complaints of school difficulties}

\author{
Eloisa Sartori Franco ${ }^{1}$, Ivone Panhoca ${ }^{2}$
}

\begin{abstract}
RESUMO
Objetivo: Estudar os sintomas vestibulares em crianças com queixas de dificuldades escolares. Métodos: Foram estudadas 88 crianças entre sete e 12 anos, que freqüentavam escolas públicas da cidade de Piracicaba no período de 2004 a 2006. Os procedimentos utilizados foram: a anamnese; exame otorrinolaringológico; exame audiológico e questionário dirigido utilizado como instrumento de coleta de dados. Resultados: Das crianças avaliadas 51\% não relataram dificuldades escolares e $49 \%$ referiram ter dificuldades escolares. A queixa referida mais comum foi a de vertigem $(22,7 \%)$, e os sintomas referidos mais comuns no ambiente escolar foram de ansiedade $(95,5 \%)$ e cefaléia $(53,4 \%)$, as dificuldades escolares mais citadas foram a de ler $(56,8 \%)$ e a de copiar $(43,2 \%)$. Conclusões: A queixa de tontura e as dificuldades em ler e copiar apresentaram relação estatisticamente significante nas crianças com queixas de dificuldades escolares.
\end{abstract}

Descritores: Doenças vestibulares; Doenças do labirinto; Vertigem/complicações; Aprendizagem

\section{INTRODUÇÃO}

Parte das queixas relatadas na clínica pediátrica, neurológica, neuropsicológica e fonoaudiológica infantil referem-se a alterações no processo de aprendizagem e/ou atraso na aquisição da linguagem ${ }^{(1)}$. Altas taxas de reprovações em escolares que ingressam no primeiro ciclo têm despertado a atenção dos especialistas que atendem crianças em idade escolar ${ }^{(2)}$.

Em um estudo relevante, 103 crianças com e sem dificuldades escolares foram avaliadas sendo que, 60 crianças $(58,2 \%)$ não relataram dificuldades e 43 crianças $(41,7 \%)$ relataram dificuldades escolares ${ }^{(3)}$.

De acordo com estudos recentes, o aprendizado pode ser afetado por fatores genéticos, afecções sensoriais periféricas, afecções neurogênicas, afecções médicas gerais, desvantagem sócio-cultural e transtornos pedagógicos ${ }^{(2)}$.

Trabalho realizado no Curso de Fonoaudiologia da Faculdade de Ciências da Saúde da Universidade Metodista de Piracicaba - UNIMEP - Piracicaba (SP), Brasil, pelo Programa de Pós Graduação em Saúde da Criança e do Adolescente do Departamento de Pediatria da Faculdade de Ciências Médicas da Universidade Estadual de Campinas - UNICAMP - Campinas (SP), Brasil. (1) Pós-graduanda do Programa de Pós-Graduação em Saúde da Criança e do Adolescente do Departamento de Pediatria da Faculdade de Ciências Médicas da Universidade Estadual de Campinas - UNICAMP - Campinas (SP), Brasil; Professora do Curso de Fonoaudiologia da Universidade Metodista de Piracicaba - UNIMEP - Piracicaba (SP), Brasil.

(2) Doutora, Professora da Faculdade de Fonoaudiologia da Pontifícia Universidade Católica de Campinas - PUCCAMP - Campinas (SP), Brasil; Orientadora do Programa Saúde da Criança e do Adolescente do Departamento de Pediatria da Faculdade de Ciências Médicas da Universidade Estadual de Campinas - UNICAMP - Campinas (SP), Brasil.

Endereço para correspondência: Eloísa Sartori Franco. R. Aldeia Campista,

103, Condomínio San Conrado, Sousas, Campinas - SP, CEP: 13104-051.

E-mail: esfranco@unimep.br

Recebido em: 1/11/2007; Aceito em: 8/9/2008
Dentro das perturbações neurogênicas que podem produzir alterações no aprendizado, temos os transtornos das integrações funcionais básicas (visomotora e auditivo-visual), do esquema corporal, da orientação espacial, do ritmo, da coordenação motora fina, da lateralidade, da função análise e síntese, da integração das partes em um todo, da simbolização, da linguagem em seus diferentes aspectos, dos impulsos, da atenção e da memória ${ }^{(4)}$.

O equilíbrio estático é considerado por pesquisadores uma função neurológica importante para a manutenção de posturas adequadas, imprescindíveis no ato de aprender. $\mathrm{O}$ equilíbrio dinâmico, também é considerado pelos autores uma função evolutiva, proporcionando indicativos de maturidade neurológica. Pesquisas relatam que crianças com imaturidade desta função têm mais probabilidade de apresentar dificuldades de aprendizado $^{(5)}$.

O sistema proprioceptivo vestibular controla a posição do corpo, os movimentos dos olhos e a percepção espacial. É por meio dele que o ser humano inicia o contato com o meio circundante. A postura, o equilíbrio físico e a coordenação motora são considerados essenciais para a aprendizagem, inclusive da linguagem falada e escrita ${ }^{(6)}$.

A disfunção vestibular infantil pode afetar consideravelmente a habilidade de comunicação, o estado psicológico e o desempenho escolar, sendo importante lembrar que o baixo rendimento escolar também pode ser um indício valioso de possível labirintopatia ${ }^{(7)}$.

$\mathrm{O}$ reconhecimento precoce das vestibulopatias infantis e o seu tratamento etiológico são essenciais na prevenção das complicações que freqüentemente decorrem no desenvolvimento motor e na aquisição de linguagem. Deve-se fazer o exame vestibular em toda a criança que apresentar uma forte 
suspeita de disfunção vestibular, muito embora não seja fácil obter da criança ou de seus pais uma descrição precisa dos sintomas $^{(8)}$.

Vertigens e outras tonturas, náuseas, vômitos, quedas, desequilíbrios ou desvios de marcha, cefaléia ou mal-estar indefinido, associado ou não à distorção visual, cansaço excessivo, mudança súbita de comportamento, agitação, perturbação do sono e medo de altura podem levar à suspeita de comprometimento do sistema vestibular. ${ }^{(7)}$

Pesquisadores aplicaram um questionário em mais de dois mil escolares. Dentre esses, 314 crianças relataram tontura há mais de um ano. $\mathrm{O}$ índice de ausência escolar durante o ano letivo foi maior nessas crianças e foi atribuído ao mal estar provocado pelas crises vertiginosas ${ }^{(9)}$.

Avaliações vestibulares foram realizadas em 29 crianças em idade escolar e 20,7\% delas, apresentaram alterações vestibulares, tendo como principais queixas: cefaléia $(48,2 \%)$; ansiedade $(44,8 \%)$; dificuldade de leitura $(34,4 \%)$; dificuldade de compreender (24,1\%); dificuldade de concentração $(37,9 \%)$ e desconforto ao brincar no gira-gira $(44,8 \%)^{(10)}$.

Pesquisas atuais mostraram os principais sintomas otorrinolaringológicos em escolares, e a tontura foi a queixa mais freqüente $(26,0 \%)$. Para os autores desta pesquisa, a dificuldade na aquisição de habilidades escolares e no desenvolvimento da linguagem, pode estar relacionada com essa queixa, levando ao questionamento sobre a necessidade de rever o que realmente pode estar atrapalhando o desenvolvimento desses estudantes ${ }^{(11)}$.

Segundo estudos, $85 \%$ das tonturas são causadas por distúrbios do sistema vestibular. Os sintomas neurovegetativos, como náuseas, vômitos, sudorese, e algumas vezes diarréia, são relatados por muitos portadores dessas alterações vestibulares $^{(12)}$.

Alterações vestibulares foram pesquisadas em crianças enxaquecosas, observando que $47 \%$ delas apresentavam queixas no desempenho escolar, tendo como principais sintomas: tontura $(72,0 \%)$; náuseas $(81,0 \%)$ e vertigem $(54,0 \%)$. Os autores constataram que os distúrbios vestibulares podem estar associados às dificuldades escolares e motoras ${ }^{(13)}$.

Os critérios para identificar afecção labiríntica na criança, descritos por estudos recentes são: a criança não gostar de ser movimentada; atraso na escolaridade; quedas freqüentes; náuseas e vômitos inexplicáveis; dificuldades na aquisição de linguagem oral e escrita; antecedentes de enxaqueca; uso de ototóxicos e otite média aguda recorrente ${ }^{(14)}$.

As dificuldades para realizar movimentos coordenados e a noção imprecisa da posição espacial de si mesmo e dos objetos, dificultam a aprendizagem em crianças com labirintopatias. A falta de aptidão para praticar alguns movimentos físicos, a adoção de posições cefálicas anormais durante a escrita, as sensações distorcidas do tamanho, do peso e da estrutura corporal, da dimensão dos objetos distantes ou das relações espaciais prejudicam o adequado contato da criança com o meio ambiente, desfavorecendo seu desenvolvimento físico e psíquico ${ }^{(8)}$.

As tonturas são freqüentes, porém às vezes são bem toleradas pelas crianças e, tal queixa só aparece mediante questionamento direcionado. Tais crianças, normalmente, são inquietas, devido à constante procura de posições de conforto e segurança, o que leva à dificuldade de concentração e à dispersão. Podem não gostar de brincar, andar de bicicleta (ou de tirar o apoio das rodas laterais), de andar sobre o muro ou guia de sarjeta, de pular corda ou amarelinha, de usar os brinquedos de playground $^{(15)}$.

Queixas inespecíficas como mudança súbita de comportamento, agitação, perturbação do sono, cefaléia, medo de altura, medo de "escuro", quedas, náuseas e vômitos, devem ser valorizadas (além dos clássicos sintomas labirínticos: tonturas rotatórias ou não; disacusia; zumbido; plenitude auricular; intolerância a sons intensos e etc.) já que podem ser evidências de problemas que comprometerão o rendimento escolar da criança e todo o processo de desenvolvimento. Esses sintomas são decorrentes de distúrbios do sistema vestibular e suas relações com o sistema nervoso central, visão, propriocepção e vários outros órgãos e sistemas localizados muitas vezes à distância do labirinto ${ }^{(8)}$.

Segundo pesquisas na área, a vertigem paroxística benigna é uma das labirintopatias infantis mais freqüentes e caracteriza-se por surtos de tontura e ou alteração de equilíbrio, comumente relacionadas com déficit do desenvolvimento da linguagem, distúrbios de comportamento psicológico e baixo rendimento escolar ${ }^{(8)}$.

Acompanhar a professora, em seu campo visual, na sala de aula, fazer cópias, transcrever as lições escritas na lousa, ler as lições do livro, escrever e concentrar-se são atividades que exigem integridade das funções oculomotoras e das interligações vestibulares ${ }^{(6)}$.

Portanto, a presente pesquisa teve como objetivo estudar as principais queixas de caráter vestibular das crianças em idade escolar com queixas de dificuldades escolares, tornando possível a melhor compreensão dos distúrbios vestibulares desencadeantes e seus sintomas, propiciando, como conseqüência, estudos futuros que redundem em programas de intervenção precoce.

\section{MÉTODOS}

\section{Tipo de estudo}

Estudo de caráter experimental, aprovado pelo Comitê de Ética (Processo 423/2003), realizado nas dependências da Clínica-Escola de uma Universidade do interior de São Paulo, bem como seus equipamentos.

\section{Seleção dos casos}

Foram sujeitos da amostra crianças entre sete e 12 anos, que freqüentavam escolas públicas da cidade de Piracicaba e foram convidadas a comparecer à Clínica-Escola durante os anos de 2004 a 2006.

Avaliamos separadamente dois grupos de crianças, sendo um grupo de crianças que não referiram dificuldades escolares e um grupo de crianças que referiram dificuldades escolares. Tais referências foram obtidas a partir de uma anamnese com as crianças juntamente com seus pais e/ou responsáveis, definindo-se a que grupo as crianças pertenceriam. 
Para a realização da presente pesquisa, foram convidados 100 escolares acompanhados de seus pais que, encaminhados a Clínica-Escola, foram orientados sobre a participação no estudo. Em função do critério de exclusão exigido nesta pesquisa (descrito a seguir), o grupo de sujeitos foi composto por 88 crianças.

Foi realizada uma reunião elucidativa em data, local e horário acordado, quando foi distribuído a cada participante uma carta de informação e o termo de consentimento livre e esclarecido. Todos os escolares dessa pesquisa foram agendados, após autorização dos responsáveis. Todos os sujeitos envolvidos consentiram na realização desta pesquisa e a divulgação de seus resultados conforme a Resolução 196/96.

\section{Critérios de inclusão para o grupo com queixa de dificul- dades escolares}

Foram incluídas na amostra as crianças que referiram queixas de dificuldades escolares; apresentaram limiares auditivos tonais de $500 \mathrm{~Hz}$ a $8000 \mathrm{~Hz}$, inferiores ou iguais a $15 \mathrm{~dB} \mathrm{NA}^{(16-}$ 17); timpanometria do tipo $\mathrm{A}$ e reflexos acústicos contralateral e ipsilateral presentes bilateralmente, nas freqüências de 500 $\mathrm{Hz}$ a $4000 \mathrm{~Hz}^{(18)}$.

\section{Critérios de inclusão para o grupo sem queixa de dificul- dades escolares}

Foram incluídas na amostra as crianças que não referiram queixas de dificuldades escolares; apresentaram limiares auditivos tonais de $500 \mathrm{~Hz}$ a $8000 \mathrm{~Hz}$, inferiores ou iguais a $15 \mathrm{~dB} \mathrm{NA}^{(16-17)}$; timpanometria do tipo A e reflexos acústicos contralateral e ipsilateral presentes bilateralmente, nas frequiências de $500 \mathrm{~Hz}$ a $4000 \mathrm{~Hz}^{(18)}$.

\section{Critérios de exclusão para ambos os grupos}

Foram excluídas as crianças menores de sete anos e maiores de 12 anos, e as que relataram sintomas ou alterações auditivas e visuais que pudessem comprometer os resultados desta pesquisa.

\section{Procedimentos}

\section{Anamnese}

Inicialmente, foi realizada uma anamnese com todas as crianças e seus pais, visando a obtenção de dados referentes às queixas de origem vestibular dando ênfase à presença ou não de vertigem, explorando queixas associadas, principalmente quanto aos aspectos auditivos, sintomas neurovegetativos, casos neurológicos com suspeita de comprometimento da fossa posterior.

\section{Exame otorrinolaringológico}

Com a contribuição voluntária do médico otorrinolaringologista, ambos os grupos foram submetidos a exame otorrinolaringológico com o objetivo de afastar a hipótese de qualquer distúrbio de orelha, nariz e garganta que pudesse repercutir sobre o sistema auditivo e vestibular.

\section{Exame audiológico}

A avaliação audiológica consistiu na realização da au- diometria tonal liminar por via aérea e por via óssea, quando necessário, pesquisa do índice percentual de reconhecimento de fala (IPRF), do limiar de reconhecimento de fala (LRF) e análise da imitância acústica, de acordo com os critérios de Mangabeira Albernaz et al. ${ }^{(18)}$ Para a realização da audiometria tonal e vocal, em cabina acusticamente tratada, foi utilizado o audiômetro da marca Madsen Midimate 622 e para a imitanciometria, o analisador de orelha média Madsen ZO-72.

Foram adotados os critérios de Glorig e Davis ${ }^{(16)}$ e de Mangabeira Albernaz et al. ${ }^{(17)}$ que consideram os padrões normais da audição em várias idades para a caracterização dos limites da audição normal.

Os resultados do estudo audiológico foram utilizados apenas para critério de inclusão.

\section{Questionário}

Questionário utilizado como instrumento de coleta de dados, elaborado por meio de um modelo fechado, contendo inicialmente dados pessoais relativos à criança (idade e sexo), série escolar, relato de dificuldades escolares, bem como histórico de retenção (Anexo 1).

Para obter informações referentes aos sintomas vestibulares, foram inseridas questões relativas aos sintomas vestibulares freqüentes; inabilidade para atividades físicas específicas; sintomas vestibulares ocorridos no ambiente escolar; dificuldades específicas na aprendizagem.

Os aspectos neurológicos foram abordados conhecendo-se o histórico de internação prolongada, manutenção contínua de medicamentos ou mesmo ingestão recente.

Para facilitar a obtenção das informações e sua posterior análise, todos os itens relacionados às variáveis estudadas foram codificados e inseridos em um banco de dados.

\section{Método estatístico}

Para a análise dos resultados, utilizamos o teste não paramétrico: Teste de Mann-Whitney para verificar possíveis associações entre as variáveis estudadas.

Obedecemos aos limites de confiança de $95 \%$, baseados nos valores de média e desvio-padrão para as variáveis e fixamos em 0,05 ou $5 \%(\leq 0,05)$ para o nível de rejeição da hipótese de nulidade e destacaremos os valores significantes.

Usamos o programa SPSS (Statistical Package for Social Sciences) em sua versão 13.0 para obtenção dos resultados.

\section{RESULTADOS}

Na Tabela 1, temos a distribuição da amostra quanto aos percentuais das queixas no desempenho escolar em função do sexo.

Na Tabela 2 apresentamos a análise comparativa da amostra em percentuais das aprendizagens específicas em função das queixas no desempenho escolar. Ao aplicar o Teste de MannWhitney, para as diferenças, notamos que as dificuldades em "ler" ( $p<0,001)$ e "copiar" ( $p<0,001)$ apresentaram uma relação estatisticamente significante entre as variáveis estudadas.

Na Tabela 3 apresentamos a análise comparativa da amostra em percentuais das queixas referidas mais freqüentes 
Tabela 1. Distribuição da amostra em relação ao sexo e dificuldade escolar referida $(n=88)$

\begin{tabular}{lccc}
\hline Dificuldade & \multicolumn{2}{c}{ Sexo } & Total \\
\cline { 2 - 3 } escolar & Feminino & Masculino & \\
\hline Não & 22 & 23 & 45 \\
& $48,9 \%$ & $51,1 \%$ & $100,0 \%$ \\
Sim & 14 & 29 & 43 \\
& $32,6 \%$ & $67,4 \%$ & $100,0 \%$ \\
Total & 36 & 52 & 88 \\
& $40,9 \%$ & $59,1 \%$ & $100,0 \%$ \\
\hline
\end{tabular}

em função das queixas no desempenho escolar. Ao aplicar o Teste de Mann-Whitney, para as diferenças, apenas a queixa referida de "tontura" apresentou uma relação estatisticamente significante $(\mathrm{p}=0,047)$.

Na Tabela 4 apresentamos a análise comparativa da amostra em percentuais dos sintomas referidos mais freqüentes ocorridos na escola em função das queixas do seu desempenho. Ao aplicar o Teste de Mann-Whitney, para as diferenças, notamos que os sintomas referidos de "vômito" e "náuseas" apresentaram uma relação estatisticamente significante $(\mathrm{p}=0,029$ e $\mathrm{p}=0,012$ respectivamente).

$p=0,121$

Tabela 2. Análise comparativa da amostra em percentuais das aprendizagens específicas em função da dificuldade escolar referida $(n=88)$

\begin{tabular}{|c|c|c|c|c|c|c|c|c|c|}
\hline \multirow{3}{*}{$\begin{array}{l}\text { Dificuldade } \\
\text { escolar }\end{array}$} & \multicolumn{8}{|c|}{ Aprendizagens específicas } & \multirow{3}{*}{$\begin{array}{c}\text { Total por } \\
\text { dificuldades }\end{array}$} \\
\hline & \multicolumn{2}{|c|}{ Ler } & \multicolumn{2}{|c|}{ Copiar } & \multicolumn{2}{|c|}{ Concentração } & \multicolumn{2}{|c|}{ Visão embaralhada } & \\
\hline & Sim & Não & Sim & Não & Sim & Não & Sim & Não & \\
\hline \multirow[t]{2}{*}{ Não } & 13 & 32 & 6 & 39 & 7 & 38 & 0 & 45 & 45 \\
\hline & $28,9 \%$ & $71,1 \%$ & $13,3 \%$ & $86,7 \%$ & $15,6 \%$ & $84,4 \%$ & $0,0 \%$ & $100,0 \%$ & $100,0 \%$ \\
\hline \multirow[t]{2}{*}{$\operatorname{Sim}$} & 37 & 6 & 32 & 11 & 3 & 40 & 2 & 41 & 43 \\
\hline & $86,0 \%$ & $14,0 \%$ & $74,4 \%$ & $25,6 \%$ & $7,0 \%$ & $93,0 \%$ & $4,7 \%$ & $95,3 \%$ & $100,0 \%$ \\
\hline \multirow[t]{2}{*}{ Total } & 50 & 38 & 38 & 50 & 10 & 78 & 2 & 86 & 88 \\
\hline & $56,8 \%$ & $43,2 \%$ & $43,2 \%$ & $56,8 \%$ & $11,4 \%$ & $88,6 \%$ & $2,3 \%$ & $97,7 \%$ & 100,00 \\
\hline$p$-valores & \multicolumn{2}{|c|}{$<0,001 *$} & \multicolumn{2}{|c|}{$<0,001^{*}$} & \multicolumn{2}{|c|}{0,208} & \multicolumn{2}{|c|}{0,146} & \\
\hline
\end{tabular}

Teste de Mann-Whitney, para diferença entre as variáveis estudadas.

Tabela 3. Análise comparativa da amostra em percentuais de queixas mais freqüentes em função da dificuldade escolar referida ( $\mathrm{n}=88$ )

\begin{tabular}{|c|c|c|c|c|c|c|c|c|c|c|c|c|c|}
\hline \multirow{3}{*}{$\begin{array}{l}\text { Dificuldade } \\
\text { escolar }\end{array}$} & \multicolumn{12}{|c|}{ Queixas mais freqüentes } & \multirow{3}{*}{$\begin{array}{c}\text { Total por } \\
\text { queixa }\end{array}$} \\
\hline & \multicolumn{2}{|c|}{ Tontura } & \multicolumn{2}{|c|}{ Atordoação } & \multicolumn{2}{|c|}{ Oscilação } & \multicolumn{2}{|c|}{ Vertigem } & \multicolumn{2}{|c|}{ Zumbido } & \multicolumn{2}{|c|}{ Instabilidade } & \\
\hline & Sim & Não & $\operatorname{Sim}$ & Não & Sim & Não & Sim & Não & Sim & Não & Sim & Não & \\
\hline \multirow[t]{2}{*}{ Não } & 5 & 40 & 1 & 44 & 0 & 45 & 10 & 35 & 6 & 39 & 4 & 41 & 45 \\
\hline & $11,1 \%$ & $88,9 \%$ & $2,2 \%$ & $97,8 \%$ & $0,0 \%$ & $100,0 \%$ & $22,2 \%$ & $77,8 \%$ & $13,3 \%$ & $86,7 \%$ & $8,9 \%$ & $91,1 \%$ & $100,0 \%$ \\
\hline \multirow[t]{2}{*}{ Sim } & 12 & 31 & 4 & 39 & 3 & 40 & 10 & 33 & 8 & 35 & 5 & 38 & 43 \\
\hline & $27,9 \%$ & $72,1 \%$ & $9,3 \%$ & $90,7 \%$ & $7,0 \%$ & $93,0 \%$ & $23,3 \%$ & $76,7 \%$ & $18,6 \%$ & $81,4 \%$ & $11,6 \%$ & $88,4 \%$ & $100,0 \%$ \\
\hline \multirow[t]{2}{*}{ Total } & 17 & 71 & 5 & 83 & 3 & 85 & 20 & 68 & 14 & 74 & 9 & 79 & 88 \\
\hline & $19,3 \%$ & $80,7 \%$ & $5,7 \%$ & $94,3 \%$ & $3,4 \%$ & $96,6 \%$ & $22,7 \%$ & $77,3 \%$ & $15,9 \%$ & $84,1 \%$ & $10,2 \%$ & $89,8 \%$ & $100,0 \%$ \\
\hline p-valores & \multicolumn{2}{|c|}{$0,047^{*}$} & \multicolumn{2}{|c|}{0,154} & \multicolumn{2}{|c|}{0,073} & \multicolumn{2}{|c|}{0,908} & \multicolumn{2}{|c|}{0,502} & \multicolumn{2}{|c|}{0,673} & \\
\hline
\end{tabular}

Teste de Mann-Whitney, para diferença entre as variáveis estudadas.

Tabela 4. Análise comparativa da amostra em percentuais dos sintomas mais freqüentes na escola em função da dificuldade escolar referida $(\mathrm{n}=88)$

\begin{tabular}{|c|c|c|c|c|c|c|c|c|c|c|c|c|c|}
\hline \multirow{3}{*}{$\begin{array}{l}\text { Dificuldade } \\
\text { escolar }\end{array}$} & \multicolumn{12}{|c|}{ Sintomas mais freqüentes na escola } & \multirow{3}{*}{$\begin{array}{r}\text { Total po } \\
\text { - sintoma }\end{array}$} \\
\hline & \multicolumn{2}{|c|}{ Cefaléia } & \multicolumn{2}{|c|}{ Ansiedade } & \multicolumn{2}{|c|}{ Otalgia } & \multicolumn{2}{|c|}{ Vômito } & \multicolumn{2}{|c|}{ Náuseas } & \multicolumn{2}{|c|}{ Tontura } & \\
\hline & Sim & Não & Sim & Não & Sim & Não & Sim & Não & Sim & Não & Sim & Não & \\
\hline \multirow[t]{2}{*}{ Não } & 22 & 23 & 42 & 3 & 6 & 39 & 3 & 42 & 7 & 38 & 6 & 39 & 45 \\
\hline & $48,9 \%$ & $51,1 \%$ & $93,3 \%$ & $6,7 \%$ & $13,3 \%$ & $86,7 \%$ & $6,7 \%$ & $93,3 \%$ & $15,6 \%$ & $84,4 \%$ & $13,3 \%$ & $86,7 \%$ & $100,0 \%$ \\
\hline \multirow[t]{2}{*}{ Sim } & 25 & 18 & 42 & 1 & 8 & 35 & 10 & 33 & 17 & 26 & 7 & 36 & 43 \\
\hline & $58,1 \%$ & $41,9 \%$ & $97,7 \%$ & $2,3 \%$ & $18,6 \%$ & $81,4 \%$ & $23,3 \%$ & $76,7 \%$ & $39,5 \%$ & $60,5 \%$ & $16,3 \%$ & $83,7 \%$ & $100,0 \%$ \\
\hline \multirow[t]{2}{*}{ Total } & 47 & 41 & 84 & 4 & 14 & 74 & 13 & 75 & 24 & 64 & 13 & 75 & 88 \\
\hline & $53,4 \%$ & $46,6 \%$ & $95,5 \%$ & $4,5 \%$ & $15,9 \%$ & $84,1 \%$ & $14,8 \%$ & $85,2 \%$ & $27,3 \%$ & $72,7 \%$ & $14,8 \%$ & $85,2 \%$ & 100,00 \\
\hline p-valores & \multicolumn{2}{|c|}{0,387} & \multicolumn{2}{|c|}{0,331} & \multicolumn{2}{|c|}{0,502} & \multicolumn{2}{|c|}{0,029 * } & \multicolumn{2}{|c|}{0,012 * } & \multicolumn{2}{|c|}{0,699} & \\
\hline
\end{tabular}

Teste de Mann-Whitney, para diferença entre as variáveis estudadas. 


\section{DISCUSSÃO}

Em nossos resultados, temos a distribuição da amostra quanto aos percentuais do desempenho escolar em função do sexo. Nenhuma diferença significante entre os dois sexos foi observada já que houve uma semelhança $(\mathrm{p}=0,121)$ entre as variáveis estudadas.

Nesses resultados notamos que, das 88 crianças avaliadas, $45(51,0 \%)$ não relataram dificuldades na aprendizagem escolar e $43(49,0 \%)$ referiram ter dificuldades na aprendizagem escolar.

Observamos dados semelhantes aos estudos de pesquisado$\mathrm{res}^{(3)}$ que avaliaram crianças com e sem dificuldades escolares sendo que, 60 crianças $(58,2 \%)$ não relataram dificuldades escolares e 43 crianças $(41,7 \%)$ relataram dificuldades escolares e aos estudos descritos anteriormente ${ }^{(13)}$ em que, os autores, ao pesquisarem alterações vestibulares em crianças enxaquecosas, observaram que $47 \%$ delas apresentavam queixas no desempenho escolar.

Deve-se levar em consideração os estudos recentes que afirmaram que o aprendizado pode ser afetado por fatores genéticos, afecções sensoriais periféricas, afecções neurogênicas, entre outras, e que dentro das perturbações neurogênicas temos os transtornos das integrações funcionais básicas, do esquema corporal e da orientação espacial ${ }^{(2,4)}$.

Autores argumentaram em seu trabalho que o equilíbrio estático e o dinâmico são funções neurológicas importante para a manutenção de posturas adequadas, imprescindíveis no ato de aprender. Em seu estudo, considerou que crianças com imaturidade destas funções têm mais probabilidade de apresentar dificuldades de aprendizado ${ }^{(5)}$.

$\mathrm{Na}$ análise comparativa da amostra em percentuais das aprendizagens específicas em função das queixas no desempenho escolar, pudemos notar que as dificuldades em "ler" $(\mathrm{p}<0,001)$ e "copiar" $(\mathrm{p}<0,001)$ apresentaram uma relação estatisticamente significante.

Das 88 crianças que participaram deste estudo, 50 crianças $(56,8 \%)$ relataram dificuldade na leitura, sendo que delas, 37 $(86,0 \%)$ referiram dificuldades na aprendizagem escolar. Das 88 crianças que participaram deste estudo, 38 crianças $(43,2 \%)$ relataram dificuldade em copiar, sendo que delas, $32(74,4 \%)$ referiram dificuldades na aprendizagem escolar.

Dados relevantes foram observados em demais trabalhos que obtiveram como principais queixas a dificuldade de leitura $(34,4 \%)$, a dificuldade de compreender $(24,1 \%)$, a dificuldade de concentração $(37,9 \%)$ ao realizarem avaliações vestibulares em crianças em idade escolar concluindo, assim, que $20,7 \%$ delas apresentaram alterações vestibulares ${ }^{(10)}$.

Pudemos constatar em nossos resultados, por meio dos percentuais das queixas referidas mais comuns entre as crianças estudadas que a queixa referida de "tontura" apresentou uma diferença estatisticamente significante $(\mathrm{p}=0,047)$ entre as crianças com queixas de dificuldades escolares e as crianças sem queixas de dificuldades escolares.

Segundo pesquisadores, $85 \%$ das tonturas são causadas por distúrbios do sistema vestibular. Os sintomas neurovegetativos como náuseas, vômitos, sudorese e, algumas vezes, diarréia são relatados por muitos portadores dessas alterações vestibulares ${ }^{(12)}$.
Os dados encontrados aproxima-se do posicionamento de estudiosos no qual afirmaram que a vertigens e outras tonturas, náuseas, vômitos, quedas, desequilíbrios entre outros, podem levar à suspeita de comprometimento do sistema vestibular $^{(6)}$.

Com base nesta análise, pudemos observar que, das 88 crianças estudadas, 17 crianças $(19,3 \%)$ queixaram-se de tontura, sendo que 12 crianças $(70,5 \%)$ referiram dificuldades na aprendizagem escolar.

Esses achados são compatíveis com estudos descritos anteriormente sobre os principais sintomas otorrinolaringológicos observados em escolares. Os autores constataram que a tontura foi a queixa mais frequiente $(26,0 \%)$ entre os estudantes. Para os autores as dificuldades na aquisição de habilidades escolares e no desenvolvimento da linguagem podem estar relacionadas com essa queixa ${ }^{(11)}$.

Dados semelhantes foram encontrados nos estudos da área em que, os autores, ao pesquisarem alterações vestibulares em crianças enxaquecosas observaram que os principais sintomas associados à enxaqueca foram além de náuseas (81\%), e vertigem $(54 \%)$, a tontura (72\%). Constataram que os distúrbios vestibulares podem estar associados às dificuldades escolares e motoras ${ }^{(13)}$.

Em nosso estudo pudemos demonstrar os percentuais dos sintomas referidos mais comuns no ambiente escolar entre as crianças estudadas. Pudemos notar que os sintomas referidos de "vômito" e "náuseas" apresentaram uma relação estatisticamente significante $(\mathrm{p}=0,029$ e $\mathrm{p}=0,012$ respectivamente) entre as crianças com dificuldades escolares e as crianças sem dificuldades escolares.

A literatura relata alguns critérios para identificar disfunção labiríntica na criança e dentre eles estão: náuseas e vômitos inexplicáveis; dificuldades na aquisição de linguagem oral e escrita; antecedentes de enxaqueca ${ }^{(14)}$.

Por meio desta análise pudemos observar que das 88 crianças estudadas, 47 crianças $(53,4 \%)$ referiram sintoma de cefaléia, sendo que dessas, 25 crianças $(53,1 \%)$ relataram dificuldades na aprendizagem escolar. Mesmo não apresentando uma relação estatisticamente significante entre as variáveis os dados mostraram um índice bastante elevado deste sintoma.

Tais fatos podem sugerir que há alteração no sistema vestibular como lembra estudos recentes que definiram que náuseas, cefaléia ou mal-estar indefinido, entre outras, podem levar à suspeita de comprometimento do sistema vestibular ${ }^{(7)}$.

Estudos vestibulares realizados em crianças em idade escolar, demonstraram a prevalência de queixas de cefaléia nas crianças avaliadas $(48,2 \%)$, valorizando os dados encontrados nesta pesquisa ${ }^{(10)}$.

É importante considerar, com base nos estudos na área que, como conseqüência desses sintomas, temos um grande índice de ausência escolar atribuído, muitas vezes, ao mal estar provocado pelas crises vertiginosas, interferindo na qualidade da aprendizagem ${ }^{(9)}$.

Segundo a literatura científica, deve-se fazer o exame vestibular em toda a criança que apresentar uma forte suspeita de disfunção vestibular, mesmo não sendo fácil obter da criança ou de seus pais uma descrição precisa dos sintomas. Segundo os autores, a vertigem paroxística benigna é uma das labirin- 
topatias infantis mais freqüentes e caracteriza-se por surtos de tontura e ou alteração de equilíbrio, comumente relacionadas com déficit do desenvolvimento da linguagem, distúrbios de comportamento psicológico e baixo rendimento escolar ${ }^{(8)}$.

Queixas inespecíficas devem ser valorizadas já que podem ser evidências de problemas que comprometerão o rendimento escolar da criança e todo o processo de desenvolvimento.

Os dados encontrados na presente pesquisa nos aproximam da afirmação de que, para que a criança possa acompanhar a professora em seu campo visual, na sala de aula, fazer cópias, transcrever as lições escritas na lousa, ler as lições do livro, escrever, concentrar-se é imprescindível a integridade das funções vestibulares e suas interligações.
Para que aspectos obscuros não impeçam uma adequada intervenção, torna-se imprescindível o conhecimento cada vez mais aprofundado, valorizando os trabalhos na área da fonoaudiologia.

\section{CONCLUSÃO}

Na presente pesquisa, pudemos concluir que nas crianças com queixas de dificuldades escolares, a queixa referida de tontura e o sintoma referido de vômito e náuseas, apresentam uma relação estatisticamente significante em comparação com as crianças sem queixas de dificuldades escolares.

\begin{abstract}
Purpose: To study vestibular symptoms in children with complaints of school difficulties. Methods: Eighty-eight children between seven and 12 years old were studied. The subjects attended public schools from Piracicaba - SP (Brazil) during the period from 2004 to 2006. The procedures included: anamnesis, otorhinolaryngological examination, audiological examination, and a directed questionnaire used as an instrument for data gathering. Results: From the evaluated children, $51 \%$ had no complaints of school difficulties, and $49 \%$ reported school difficulties. The most common complaint reported was vertigo (22.7\%), and the most common symptoms reported in school environment were anxiety (95.5\%) and chronic headache (53.4\%). The most cited school difficulties were reading (56.8\%) and copying (43.2\%). Conclusions: Vertigo complaints and reading and copying difficulties presented a statistically significant relation in children with complaints of school difficulties.
\end{abstract}

Keywords: Vestibular diseases; Labyrinth diseases; Vertigo/complications; Learning

\section{REFERÊNCIAS}

1. Schirmer CR, Fontoura DR, Nunes ML. Distúrbios da aquisição da linguagem e da aprendizagem. J Pediatr (RJ). 2004;80(2 Supl):S95-S103.

2. Schachter DC, Schachter DC, Pless IB, Bruck M. Self-report of family histories of learning difficulties. Can J Psychiatry. 1992;37(1):29-32.

3. Farias LS; Toniolo, IF; Cóser, PL. P300: avaliação eletrofisiológica da audição em crianças sem e com repetência escolar. Rev Bras Otorrinolaringol. 2004;70(2):194-9.

4. Sandler AD, Watson TE, Footo M, Levine MD, Coleman WL, Hooper SR. Neurodevelopmental study of writing disorders in middle childhood. J Dev Behav Pediatr. 1992;13(1):17-23.

5. Guardiola A, Ferreira LT, Rotta NT. Associação entre desempenho das funções corticais e alfabetização em uma amostra de escolares de primeira série de Porto Alegre. Arq Neuropsiquiatr. 1998;56(2):281-8.

6. Ganança MM, Caovilla HH. Labirintopatias na infância. In: Caldas N, Sih T. Otologia e audiologia em pediatria. São Paulo: Revinter; 1999. p.277-86.

7. Campos MI, Ganança FF, Caovilla HH, Ganança MM. Prevalência de sinais de disfunção vestibular em crianças com vertigem e/ou outros tipos de tontura. RBM-ORL. 1996;3(3):165-70.

8. Caovilla HH, Ganança MM, Munhoz MS, Silva ML, Ganança FF, Frazza MM. Vertigem paroxística benígna da infância. In: Silva ML, Munhoz MS, Ganança MM, Caovolla HH. Quadros clínicos otoneurológicos mais comuns. São Paulo: Atheneu; 2000. p.109-17.

9. Russell G, Russell G, Abu-Arafeh I. Paroxysmal vertigo in children--an epidemiological study. Int J Pediatr Otorhinolaryngol. 1999;49 Suppl 1:S105-7.
10. Franco ES, Caetanelli EB. Avaliação vestibular em crianças sem queixas auditivas e vestibulares, por meio da vectonistagmografia computadorizada. Arq Int Otorrinolaringol. 2006;10(1):46-54.

11. Araujo AS, Moura JR, Camargo LA. Principais sintomas otorrinolaringológicos em escolares. Arq Otorrinolaringol. 2004;8(1):524.

12. Lourenço EA, Lopes KC, Pontes Jr A, Oliveira MH, Umemura A, Vargas AL. Distribuição dos achados otoneurológicos em pacientes com disfunção vestíbulo-coclear Rev Bras Otorrinolaringol. 2005;71(3):28896.

13. Narciso AR, Zeigelboim BS, Alvarenga KF, Jacob L, Costa Filho AO, Ribas A. Alterações vestibulares em crianças enxaquecosas. Arq Otorrinolaringol. 2004;8(3):201-6.

14. Lavinsky L, Abelin CA, D’Avila C, Lavinsky M. Exame otoneurológico na infância. In: Caldas N, Sih T. Otologia e audiologia em pediatria. São Paulo: Revinter; 1999. p.287-95.

15. Formigoni LG. A avaliação vestibular na criança. In: Ganança MM. Vertigem tem cura? São Paulo: Lemos; 1998. p.117-26.

16. Glorig A, Davis H. Age, noise and hearing loss. Ann Otol Rhinol Laryngol. 1961;70:556-71.

17. Mangabeira-Albernaz P, Mangabeira-Albernaz PL, MangabeiraAlbernaz LG, Mangabeira-Albernaz Filho P. Otorrinolaringologia prática. 10a ed. São Paulo: Sarvier; 1981.

18. Mangabeira-Albernaz PL, Ganança MM, Caovilla HH, Ito YI, Novo NF, Juliano Y. Aspectos clínicos e terapêuticos das vertigens. Acta AWHO. 1986;5(2 Supl):49-109. 
Anexo 1. Anamnese

Nome:

Idade:

D.N.:

Nome do Responsável:

Telefone de contato:

Escolaridade:

Histórico de repetência:

Escola:

Seu filho queixa-se:

$\square$ Nega tontura

$\square$ Tontura rotatória

Seu filho queixa-se:

$\square$ Nega perda auditiva

$\square$ Em O. E.

Seu filho queixa-se:

$\square$ Nega zumbido

$\square$ Em O. E.

$\square$ Em O.D.

$\square$ Em A.O.

Seu filho sente freqüentemente:

$\square$ Vertigem
$\square$ Tontura
$\square$ Náuseas
$\square$ Cefaléia
$\square$ Palidez
$\square$ Ansiedade
$\square$ Vômitos
$\square$ Depressão
$\square$ É estabanado

Seu filho não gosta de:

$\square$ Ser movimentado

$\square$ Altura

$\square$ Andar de bicicleta

Seu filho tem:

$\square$ Dificuldade de concentração

$\square$ Dificuldade de ler

$\square$ Dificuldade de copiar
Anamnese - Otoneurológica

Tontura

Tontura não rotatória

Flutuação

$\square$ Instabilidade

Atordoação

Perda auditiva

Em O.D.

Em A.O.

Flutuante

Zumbido

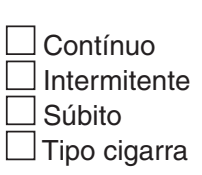

$\square$ Tipo cachoeira

$\square$ Tipo panela de pressão

$\checkmark$ Pulsátil

Sintom as

Sudorese

Dor no ouvido

Taquicardia

Hipoacusia

Hiperacusia

$\square$ Zumbido

Fobias

Plenitude auricular

$\mathrm{Habilidades}$

Amarelinha

Gira - gira

Pular corda

$\mathrm{Na}$ escola

Visão embaralhada

Posição de cabeça anormal durante a escrita

Dificuldade de interagir $\square$ Dificuldade de entender

$\square$ Sensação de desligamento

$\square$ Escurecimento da visão

Atordoação

$\square$ Sensação de cabeça oca

$\square$ Líquido no ouvido

Mal estar indefinido

Cai com freqüência

\section{Observação:}

Já tomou medicamentos por um longo tempo?

Toma medicamento atualmente?

Já foi internado? Por quê? 